\title{
Slowly-Varying Microwave Emissions from the Solar Corona
}

\author{
By KEN F. TAPPING AND KAREN L. HARVEY \\ ${ }^{1}$ Dominion Radio Astrophysical Observatory, P.O. Box 248, Penticton, British \\ Columbia,V2A 6K3, Canada \\ ${ }^{2}$ Solar Physics Research Corporation, 4270 Calle Desecada, Tucson, AZ 85718, USA
}

Measurements of the total microwave $\dagger$ emission from the solar disc at single and multiple wavelengths provide a useful indicator of solar activity, and correlate well with other indices of solar activity and with solar luminosity.

Since activity in the solar atmosphere is driven by the emergence, redistribution and disappearance of magnetic flux, the close and linear correspondence between the microwave flux densities, as observed at 10.7 and $21 \mathrm{~cm}$ wavelengths, provide a convenient and objective indicator of solar magnetic activity.

Microwave emissions from the "non-flaring" solar corona originate in a wide range of sources. These generally lie in active regions, and comprise two main types: bright, compact sources a few arc-seconds across and brightness temperatures of the order of $10^{6} \mathrm{~K}$, and weaker, diffuse sources arc-minutes in size and having brightness temperatures of about $10^{4} \mathrm{~K}$. There is also evidence of a weaker, widespread emission covering large areas of the solar disc. The compact sources are probably due to gyroresonance, although some could be non-thermal. The diffuse widespread emission are almost certainly free-free thermal emission from plasma concentrations in the corona.

The strong correlation between the $10.7 \mathrm{~cm}$ flux and other activity indices, even when they are produced by disimilar processes and at different heights in the solar atmosphere, may at least partially be due to the overiding role played by magnetic activity in dictating the structure of the chromosphere and corona. However, the consistent correspondences over wide ranges of activity suggest dependences upon relatively few quantities and that the microwave emission from the non-flaring Sun is dominated by a single process, which is closely and simply-related to density and temperature, such as free-free (bremsstrahlung) emission from plasma concentrations trapped in coronal and chromospheric magnetic structures. Attempts to "budget" the total microwave emission at $2.8,10.7$ and $21 \mathrm{~cm}$ wavelengths suggest that this is the case.

Where ambient magnetic fields exceed $3000 / \lambda$ Gauss, gyroresonance can produce much higher optical depths than free-free emission, and accounts for the bright emission observed in the vicinity of sunspots. Although not the dominant contributor to the total emission from the solar disc, these sources stand out brightly in high-resolution maps of active regions.

To examine the assumptions of dominance of free-free thermal emission, and simple dependences upon few parameters, we compute the spectrum for a range of coronal density models, and compare the results with observations. The agreement is good, suggesting that the total disc flux is dominated by thermal, free-free emission, and that the intensity is controlled mainly by the density at the base of the corona, but is otherwise fairly tolerant of the density model used.

\section{Introduction}

The solar corona is an extended, inhomogeneous and dynamically-disturbed plasma, pervaded by magnetic fields which are strong enough to control its distribution and activity. The spatial scales of the inhomogeneities range from structures larger than the

$\dagger$ We use the term 'microwave' here to refer to electromagnetic waves in the wavelength range $1-100 \mathrm{~cm}$ 
photospheric diameter, probably down to as small as an electron gyroradius. A review of the properties of the corona and its activity is given by Koutchmy et al. (1991), and Rabin et al. (1991).

The inhomogeneity and dynamism make the corona a treasure house of radio emissions. These range from compact, bright sources having durations of milliseconds (Slottje 1978; Slottje et al. 1980; Zhao \& Jin 1981; Tapping et al. 1983) through the menagerie of transient emissions associated with flares, to the slow variations following the ebb and flow of magnetic activity inside and outside active regions, and underlying these, a base level known as the quiet Sun. The complex field of solar radio astronomy is surveyed in detail in books by Kundu (1965) and Krüger (1979).

Early solar radio telescopes had small antennas, and could only measure the integrated flux from all sources present on the solar disc. Using "characteristic timescale" as a criterion, the emission was divided, rather arbitrarily, into three components: a rapidlyvarying component associated with flares, typically having durations shorter than about an hour, a slowly-varying or $S$-component embracing all slower variability, and underlying these emissions, corresponding to an extrapolated zero level of activity, a constant, quiet Sun level. The S-component was first defined as a particular subset of solar radio emissions independently by Covington at $10.7 \mathrm{~cm}$ wavelength $(1947,1948)$ and Lehaney \& Yabsley at $21 \mathrm{~cm}(1949)$.

The emissions from the non-flaring Sun vary with the ebb and flow of activity in the solar atmosphere, and are connected with both localized and large-scale changes in the structure of the chromosphere and corona as the level of activity varies. The quiet Sun is not different from the $S$-component, it merely reflects the baseline of a continuum of states which reflect increases in coronal density and magnetic activity. The full-disc microwave fluxes are indices of the Sun's "stellar" properties, rather than the localized dynamics of individual active regions and magnetic structures, even though the sources in these contribute much of the total microwave emission from the non-flaring Sun.

Radio indices of solar activity, such as the $10.7 \mathrm{~cm}$ flux, consist of measurements of the total emission with the flare contributions filtered out. They comprise the sum of the quiet Sun and S-component emissions, with the quiet Sun contributing a constant, base level, and the variability attributed to the S-component.

Compared with solar radio emissions other than the quiet Sun component, the Scomponent has remarkably-stable properties (Krüger 1979). It is most easily observed at wavelengths between 1 and $100 \mathrm{~cm}$, and has a spectrum peaking at a wavelength of about $10 \mathrm{~cm}$. The fortuitous choice by Covington and his co-workers of $10.7 \mathrm{~cm}$ for patrol observations of the S-component has provided an effective estimate of the S-component intensity and a good index of solar activity (the $10.7 \mathrm{~cm}$ flux $\dagger F_{10.7}$ ) used both in its own right, and since it correlates well with other indices (Tapping 1987), as a proxy for other quantities which are more difficult to measure with the desired continuity.

Research on solar microwave emissions covers a wide range of topics, including: the relationship between the integrated emission and other activity indices, and with largescale structural changes in the solar atmosphere; what magnetic structures house the sources; what emission mechanisms are involved, and what are their relative importances. In this paper we will discuss these points and investigate how the integrated emission relates to large-scale structure of the solar atmosphere.

$\dagger$ what has become widely referred to as a flux is reality a flux density, having the dimensions of $W \cdot m^{-2} \cdot H z^{-1}$ 


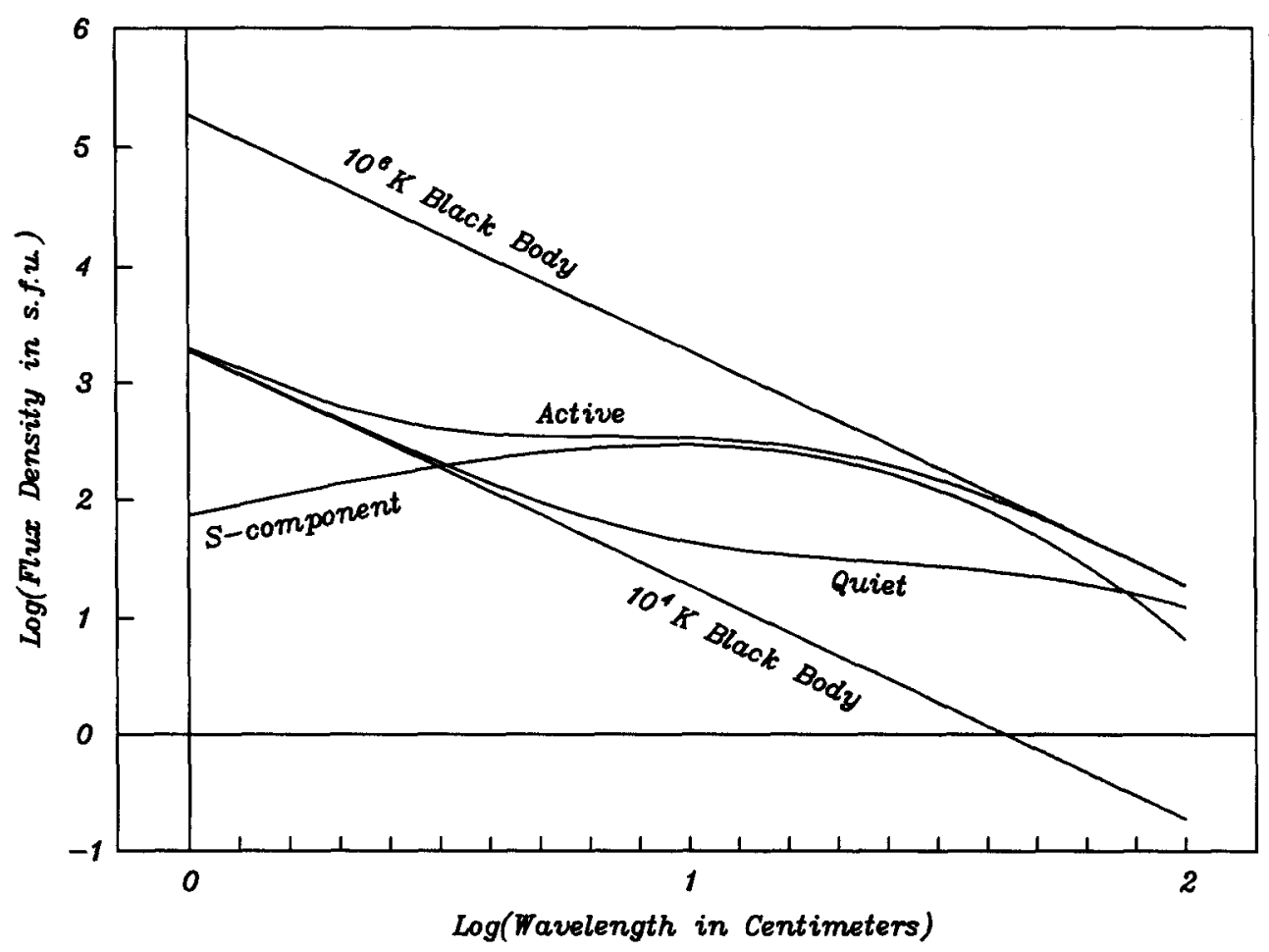

FIgURE 1. The spectrum of the integrated microwave emission from the solar disc. The S-component is the difference between the spectra of the quiet and active Sun.

\section{The total disc emission}

Figure 1 shows the spectrum of the integrated emission from the non-flaring Sun over the wavelength range $1-200 \mathrm{~cm}$. It is a composite, constructed from plots in Krüger (1979), Kundu (1965), and from a large collection of multi-wavelength flux measurements published in Aeronautica Acta (Nicolet \& Bossy 1984). In general, the spectral curve of solar radio emissions lie between the two curves marked "active" and "quiet". At activity minimum, the spectrum lies along the lower curve, marked "quiet", and at activity maximum, it approaches the upper curve.

The area between the two curves represents the S-component, the spectrum of which is estimated by subtracting the "quiet" spectrum from the spectrum of the integrated emission. The $\mathrm{S}$-component is strongest at about $10 \mathrm{~cm}$ wavelength.

In the solar corona, the contribution to the total plasma pressure by magnetic fields far exceeds that due to particle motions (i.e. $B^{2} / 2 \mu \gg N k T$ ), and the electrical conductivity is generally high. Therefore, the distribution and movements of magnetic flux usually control and support the concentrations of plasma which give rise to the S-component. This interpretation was proposed soon after the S-component was discovered (Denisse 1948; Lehaney \& Yabsley 1949).

The two straight lines represent the emission curves for black bodies at temperatures of $10^{4}$ and $10^{6} \mathrm{~K}$. The shapes of the curves suggests a thermal emission mechanism, where the $10^{4} \mathrm{~K}$ chromosphere is viewed through an attenuating, $10^{6} \mathrm{~K}$ corona:

$$
T_{b}(\lambda)=\alpha(\lambda) T_{\text {chromosphere }}+(1-\alpha(\lambda)) T_{\text {corona }}
$$




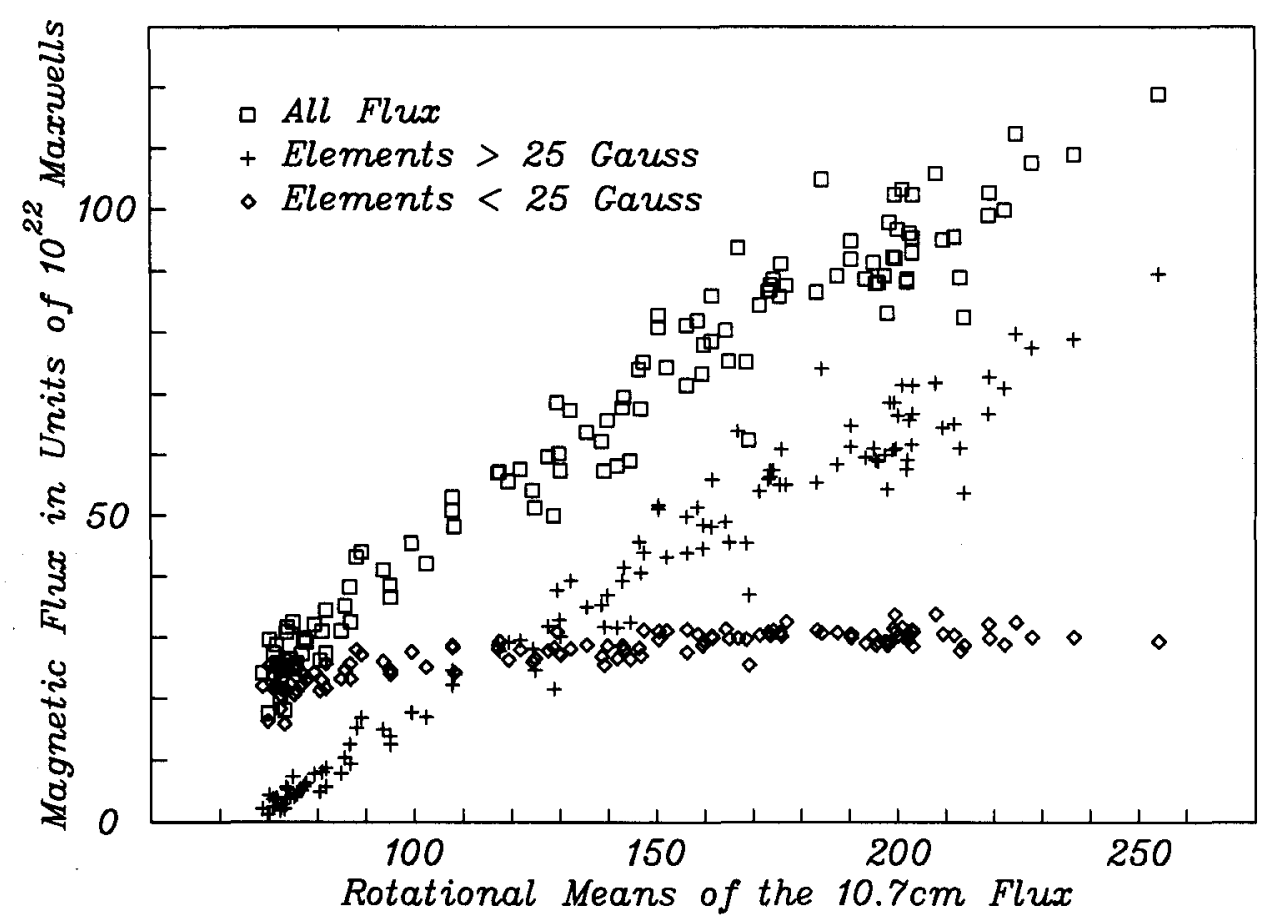

FIGURE 2. Total photospheric magnetic flux plotted against the cotemporal rotational means of the $10.7 \mathrm{~cm}$ flux for Solar Cycle 21 .

where $T_{b}$ is the observed brightness temperature, and $\alpha$ is the transmissivity of the corona. Both these quantities are functions of wavelength.

Felli et al. (1977) observed a correlation between the brightness of the emission at 2.8 $\mathrm{cm}$ wavelength and the total amount of magnetic flux, and Zwaan (1987) found a linear relationship between coronal emissions and magnetic flux in individual active regions. In general the solar photosphere can be separated into weak and strong-field areas (Rabin et al. 1991; Harvey 1992), where the average average magnetic field in the map elements is significantly weaker or stronger than about 25 Gauss. Over the whole of Cycle 21 , the magnetograph at the National Solar Observatory at Kitt Peak was used to measure the total magnetic flux over the solar sphere. Figure 2 shows a plot of total magnetic flux over the solar sphere against cotemporal rotational averages of the $10.7 \mathrm{~cm}$ flux.

The upper plot (squares) is the total magnetic flux. The lower plot (crosses) is the total magnetic flux contained in elements stronger than 25 Gauss. Subtracting the values gives the total magnetic flux in elements weaker than 25 Gauss (diamonds). The $10.7 \mathrm{~cm}$ flux correlates well with the total magnetic flux in elements stronger than 25 Gauss, but not with the magnetic flux in elements weaker than that value. Moreover, the total flux in the "weak" elements does not vary over the solar cycle, and is present even at solar minimum. According to Figure 2, the total photospheric magnetic flux contained in "weak" elements is about $2.5 \times 10^{23}$ Maxwells, corresponding to an average photospheric magnetic field strength of about 4 Gauss. Although playing no visible role in the S-component, the weak magnetic fields may play a role in maintaining the plasma distributions which produce the quiet Sun spectrum. 
The rotational means of the $10.7 \mathrm{~cm}$ flux are an excellent proxy for the total photospheric magnetic flux over the solar sphere:

$$
\Phi\left(\text { in units of } 10^{22} \text { Maxwells }\right)=a S+b
$$

where $a=0.5, b=-10$, and $S$ is the appropriate mean value of $F_{10.7}$, measured in solar flux units $\dagger$.

The strong correlation between the S-component intensity and total magnetic flux has been observed at $21 \mathrm{~cm}$ wavelength, in 11 measurements made during Cycle 22 by Burke (1993).

\section{Contributors to the total microwave flux}

The advent of high resolution radio telescopes has made it possible to map the Scomponent, firstly over the solar disc and then within individual active regions. Concentrations of S-component emission have been found in association with sunspots (Alissandrakis et al. 1980; Chiuderi Drago et al. 1982; Shibasaki et al. 1983; Alissandrakis \& Kundu 1984), bright plage (Gaizauskas \& Tapping 1980; Felli et al. 1981; Shevgaonkar \& Kundu 1985; Gaizauskas \& Tapping 1988), filaments overlying magnetic polarity reversal lines (Kundu et al. 1981; Erskine et al. 1983; Gaizauskas \& Tapping 1988), and strong magnetic field strength gradients (Alissandrakis \& Kundu 1984). However, none of these associations are consistent. Examples of all these magnetic features have been observed which are devoid of detectable microwave emission (Felli et al. 1981; Webb et al. 1983; Gaizauskas \& Tapping 1988). In addition, sources are sometimes observed where no concentrations of magnetic flux are observed (Pallavicini al. 1979; Felli et al. 1981; Kundu et al. 1981; Webb et al. 1983; Gaizauskas \& Tapping 1980).

However, despite this complexity, Pallavicini et al. (1979) show that on the basis of observations at $2.8 \mathrm{~cm}$ and in soft X-rays, the $\mathrm{S}$-component sources can be divided into two broad classes: (a) diffuse sources, having brightness temperatures of the order of $10^{4} \mathrm{~K}$ and angular sizes of arc-minutes, and (b) compact sources, with brightness temperatures of $10^{6} \mathrm{~K}$ or more and angular sizes of only a few arc-seconds. The diffuse sources are also associated with bright soft X-ray emission, whereas the compact ones are not.

Through examining full-disc maps and scans made at $2.8 \mathrm{~cm}, 10.7 \mathrm{~cm}$ and $21 \mathrm{~cm}$, Tapping (1987), and Burke (1993), identify a further contribution, a weak, widely-distributed emission which is distributed over large areas of the solar disc, and which can produce a significant contribution to the total microwave flux. From maps made at $2.8 \mathrm{~cm}$ wavelength in 1981, and at $21 \mathrm{~cm}$ in 1992 and 1993, it appears that this contribution originates in areas of "magnetic network".

\section{Emission mechanisms}

This is a short discussion only; more comprehensive treatments can be found in Kundu (1965) and Krüger (1979). Microwave emissions by the non-flaring Sun are mostly thermal, with possibly a contribution by non-thermal processes.

The thermal emission is due to the deflection of thermal electrons by interactions with ions (free-free, bremsstrahlung emission) or magnetic fields (gyroresonance). For convenience's sake, they are usually discussed as two separate processes, in the limits of "weak" and "strong" magnetic fields.

$$
\dagger 1 \text { solar flux unit (s.f.u.) }=10^{-22} W \cdot m^{-2} \cdot H z^{-1}
$$


The two distinct classes of source evident in the study by Pallavicini et al. (1979) indicate at least two emission processes. The diffuse sources, having brightness temperatures of the order of $10^{4} \mathrm{~K}$ and producing soft X-ray emission, can be explained in terms of free-free emission from density enhancements supported in the corona by active region magnetic fields.

The compact sources are brighter $\left(10^{6} \mathrm{~K}\right)$, but not producers of $\mathrm{X}$-rays. They cannot be accounted for using free-free emission models. Some of them might be non-thermal, but the majority can be accounted for in terms of gyroresonance. This process can produce bright, optically-thick thermal sources in the corona if the ambient magneiic fields are stronger than about 3000/ $\lambda$ Gauss (Kakinuma \& Swarup 1962; Zheleznyakov 1962 ), where $\lambda$ is the observing wavelength in centimeters.

\subsection{Thermal free-free emission}

The absorption coefficient for free-free interactions between thermal electrons and "heavy" ions is given by (Kundu 1965):

$$
\kappa_{f f}=\frac{a \lambda^{2} N^{2}}{n c^{2} T^{3 / 2}}
$$

where $\lambda$ is the observing wavelength, $N$ the electron density, $n$ the refractive index, $c$ the speed of light and $T$ the ambient electron temperature. The constant $a$ is $1.1 \times 10^{-11}$ in the chromosphere and $1.6 \times 10^{-11}$ in the corona.

The refractive index is:

$$
n=\left(1-\left(\frac{f_{0}}{f}\right)^{2}\right)^{\frac{1}{2}}
$$

where $f=c / \lambda$ is the observing frequency, and $f_{0}$ is the plasma frequency:

$$
f_{0}=9 \sqrt{N}(\mathrm{~Hz})
$$

We assume here that ambient magnetic fields are sufficiently weak for the electron gyrofrequency (2.8B MHz, where $\mathrm{B}$ is in Gauss) to be roughly at least an order of magnitude smaller than the observing frequency.

\subsection{Thermal gyroresonance}

Where the electron gyrofrequency is larger than about a quarter of the observing frequency, the absoprtion coefficient by gyroresonance becomes significant. The exact formula for the absorption coefficient is very complicated. However, a useful approximate form is given by Kundu (1965):

$$
\kappa_{g r}=1.5 \times 10^{-4} \Phi_{s} \frac{N}{f_{H}}\left(\frac{2 k T}{m c^{2}}\right)^{s-3 / 2} \zeta_{s}^{ \pm}(\theta) \exp \left(\frac{\left(1-s \frac{f_{H}}{f}\right)^{2}}{\frac{2 k T}{m c^{2}} \cos ^{2} \theta}\right)
$$

where

and

$$
\Phi_{s}=\frac{2}{s !}\left(\frac{s}{2}\right)^{2 s-1}
$$

$$
\zeta_{s}^{ \pm}(\theta)=(\sin \theta)^{2 s-2} \frac{\left(C_{1} \pm C_{2} \cos \theta\right)^{2}}{\cos \theta}
$$

where $N=$ electron density, $f_{H}=$ electron gyrofrequency, $f=$ observing frequency $=$ $c / \lambda, \lambda=$ observing wavelength, $c=$ speed of light, $k=$ Boltzmann's constant, $T=$ 
ambient electron temperature, $m=$ electron rest mass, $s=$ harmonic number $(2,3,4,5$ etc.), $C 1$ and $C_{2}=$ axes of polarization ellipse (where $1=C_{1}^{2}+C_{2}^{2}$ ), and $\theta=$ angle between magnetic field and line of sight.

The plus and minus signs indicate the ordinary and extraordinary wave modes. Gyroresonant emission is generally highly polarized.

\subsection{Non-thermal emission}

Bright microwave sources which are devoid of X-ray emission cannot be due to free-free thermal emission, but, if the ambient magnetic fields are strong enough, can be caused by gyroresonance. Sources having brightness temperatures of the order of $10^{6} \mathrm{~K}$ which are located in areas where the magnetic fields are estimated to be too weak for gyroresonance at that wavelength have been suggested to be non-thermal.

To drive non-thermal emission processes, such as the gyrosynchrotron mechanism, accelerated (non-thermal) electrons are needed. Where these come from is not yet clear, but Chiuderi Drago \& Melozzi (1984) have demonstrated that sources having the observed properties can be driven by gyrosynchrotron emission produced by similar populations of accelerated electrons to those believed to give rise to Type III bursts.

Non-thermal emissions are believed to be a minor contribution to the total microwave flux and will not be further discussed here.

\section{Propagation of microwaves in the solar atmosphere}

The solar atmosphere itself can affect the polarization, intensity and direction of radio waves passing through it, and even if the emissions can be detected at the Earth at all.

The absorption by free-free processes and gyroresonance attenuate the incident radio emissions and then add a thermal noise contribution from the ambient plasma, so that in passing through a thin slab of plasma, the brightness temperature of the incident waves is modified:

$$
T_{b}(n e w)=\alpha T_{b}(\text { old })+(1-\alpha) T_{0}
$$

where $\alpha$ is the transmissivity of the layer, and $T_{0}$ is the electron temperature in it.

The propagation of radio waves in the solar atmosphere is discussed in some detail by Kundu (1965), here we will just mention some of the main points. In the absence of magnetic fields, the refractive index is given by equation 4.4. Waves can only propagate when $f>f_{0}$.

In the presence of magnetic fields, the situation becomes more complicated. A wave propagating in any direction with respect to the magnetic field is split into two polarized waves, an ordinary wave and an extraordinary wave, each of which has its own refractive index and absorption coefficient. The presence of a magnetic field in the medium between an unpolarized source and the observer can lead to a polarized wave being observed. Changes in the distribution of magnetic flux in a magnetic structure which houses sources of radio emission can produce changes in both the spectrum and polarization of the emission being observed.

In general, if the frequency of the radio emissions is less than about 10 times the electron gyrofrequency in the source or in the intervening medium through which the waves are travelling, the magnetic fields are playing a significant role in their generation and propagation, and must be taken into account in any investigation of the variability of the emission. 


\section{Models for solar microwave emissions}

The gyroresonance process has been used extensively in studying emission from individual magnetic structures. However attempts to model the Sun's total microwave emission are largely based upon free-free, thermal emission (Denisse 1948; Smerd \& Westfold 1949; Christiansen \& Mathewson 1959; Chiuderi Drago et al. 1982; Tapping \& DeTracey 1990).

The two processes are usually invoked separately; the bright sources close to concentrations of magnetic flux are usually interpreted in terms of gyroresonance, and emission from elsewhere is assumed to be free-free. However, with the magnetic fields being the dominant contributor to the total pressure in the corona, it is probably more realistic (but much more complicated) to combine the two processes (Zlotnik 1968; Lantos 1968; Krüger et al. 1985). However, simple free-free models can yield useful results and within their limitations, are much more tractable.

An indication of the average relative importance of the two processes can be obtained from making a solar emission budget. At $2.8 \mathrm{~cm}$, the quiet Sun has an average disc brightness temperature of about $10,000 \mathrm{~K}$. A single compact source having an angular diameter of 10 arc-sec and a brightness temperature of $10^{6} \mathrm{~K}$ increases the brightness temperature of the disc by about $30 \mathrm{~K}$, which, if there are no other sources on the (quiet) disc, corresponds to an increase in the average brightness temperature of the disc of $0.3 \%$. In reality, such sources are unlikely to occur without sources of additional thermal, free-free emission, so the contribution by compact, gyroresonant or perhaps non-thermal sources to the total disc emission at $2.8 \mathrm{~cm}$ is likely to be small. At longer wavelengths, the quiet Sun brightness temperature increases, so the compact sources probably contribute relatively even less to the total emission.

In their estimates of the S-component budget, Tapping (1987), and Burke (1993), separate three contributions to the Sun's emission at $2.8,10.7$ and $21 \mathrm{~cm}$ wavelength: active region emission, a widely-distributed background, and the quiet Sun. After the quiet Sun component is subtracted, the contributions from active regions and the widelydistributed emission are comparable at all three wavelengths.

Apart from the contributions by compact sources, the rest of the centimetric emission from active regions is thermal bremsstrahlung, and the widely-distributed contribution is almost certainly produced by the same mechanism. The dominance of free-free emission, together with the multiplicity of sources, both inside and outside active regions, is probably the reason for the stable properties of total disc emission, and its consistent correlations with other indices of solar activity, which are produced by different processes.

\section{A thermal, free-free emission model}

Most models for the microwave emission from the non-flaring Sun are based upon the assumption that thermal free-free emission dominates other contributions. Based upon measurements or assumptions, a model for the spatial distribution of coronal density is constructed, and the resulting free-free emission intensity and spectrum calculated. One of the earliest (Waldmeier \& Müller 1950) invoked "coronal condensations", which are concentrations of plasma over active regions. The agreement between $5303 \AA$ coronagrams and maps made at $20 \mathrm{~cm}$ wavelength support this (Waldmeier 1959). Other atmosphere models, such as the Newkirk streamer model, also could produce the observed spectrum and intensity. One of the more recent attempts is by Tapping \& DeTracey (1990). This study suggested the total emission to be primarily sensitive to the density at the base of the corona, and small changes in the S-component spectrum during the solar cycle 
might be explained in terms of changes in the structure of the interface between the chromosphere and corona. However, this model, like many others, does not take into account the very significant role of coronal magnetic fields in controlling the plasma density, or else handles them empirically.

It is very interesting how a variety of density models, based upon measurements, empirical concepts or crude approximations, all account for the centimeter wavelength spectrum of the quiet and active Sun with a good degree of success. This suggests a degree of independence of the actual density model used.

\subsection{The density models}

The spatial distribution of plasma and magnetic flux in the solar atmosphere is inhomogeneous over a wide range of spatial scales and varies with time. It would be very difficult to construct an accurate distribution for any particular moment, and the validity of that distribution at a different time would not be clear. We therefore use a different approach.

We define two density models, $f_{\min }(z)$ and $f_{\max }(z)$, where $f_{\min }$ lies below all "realistic" density distributions and $f_{\max }$ lies above them. Since magnetic fields generally increase the amount of plasma supported in the solar atmosphere, $f_{\min }(z)$ is assumed to be defined by vertical balance of plasma pressures, with no magnetic fields, as in the "hydrostatic model" used by Tapping \& DeTracey (1990):

$$
N=N_{0} \exp \left(-\frac{m_{0} g_{0}}{k T} \frac{R+Z_{0}}{R+Z_{0}+z} z\right)
$$

where $k=$ Boltzmann's constant, $N=$ the ambient electron density, $N_{0}=$ the electron density at the base of the corona, $m_{0}=$ the mass of the hydrogen atom, $g_{0}=$ the gravitational acceleration at the photosphere, $R=$ the photospheric radius, $Z_{0}=$ the height of base of corona above photosphere, and $z=$ the height measured from the base of the corona.

The upper envelope model, $f_{\max }(z)$ is more difficult to estimate. However, it is extremely unlikely that magnetic fields will enhance coronal densities to the point where the density will not fall with increasing height above the photosphere. For the upperenvelope model " $f_{\max }(z)$ ", we use "uniform-density slab", $N=N_{0}$, with the density in the corona being constant with height and equal to the value at its base.

For comparison purposes, we include two density models which are based upon measurements: the Baumbach-Allen model and the Newkirk coronal streamer model, both of which have been modified to accommodate a density $N_{0}$ at the base of the corona.

The Baumbach Allen model is

$$
N=N_{0} \frac{1}{a_{1}+a_{2}}\left(a_{1} x^{6}+a_{2} x^{16}\right)
$$

where $x=R /\left(R+Z_{0}+z\right), a_{1}=1.55$ and $a_{2}=2.99$, and the scaled version of the Newkirk streamer model:

$$
N=a_{1} N_{0} \exp \left(a_{2} x\right)
$$

where $a_{1}=0.014$ and $a_{2}=4.32$.

The only degree of freedom in these models in the density at the base of the corona. They can be fixed using a single flux density or brightness temperature at a single wavelength. 

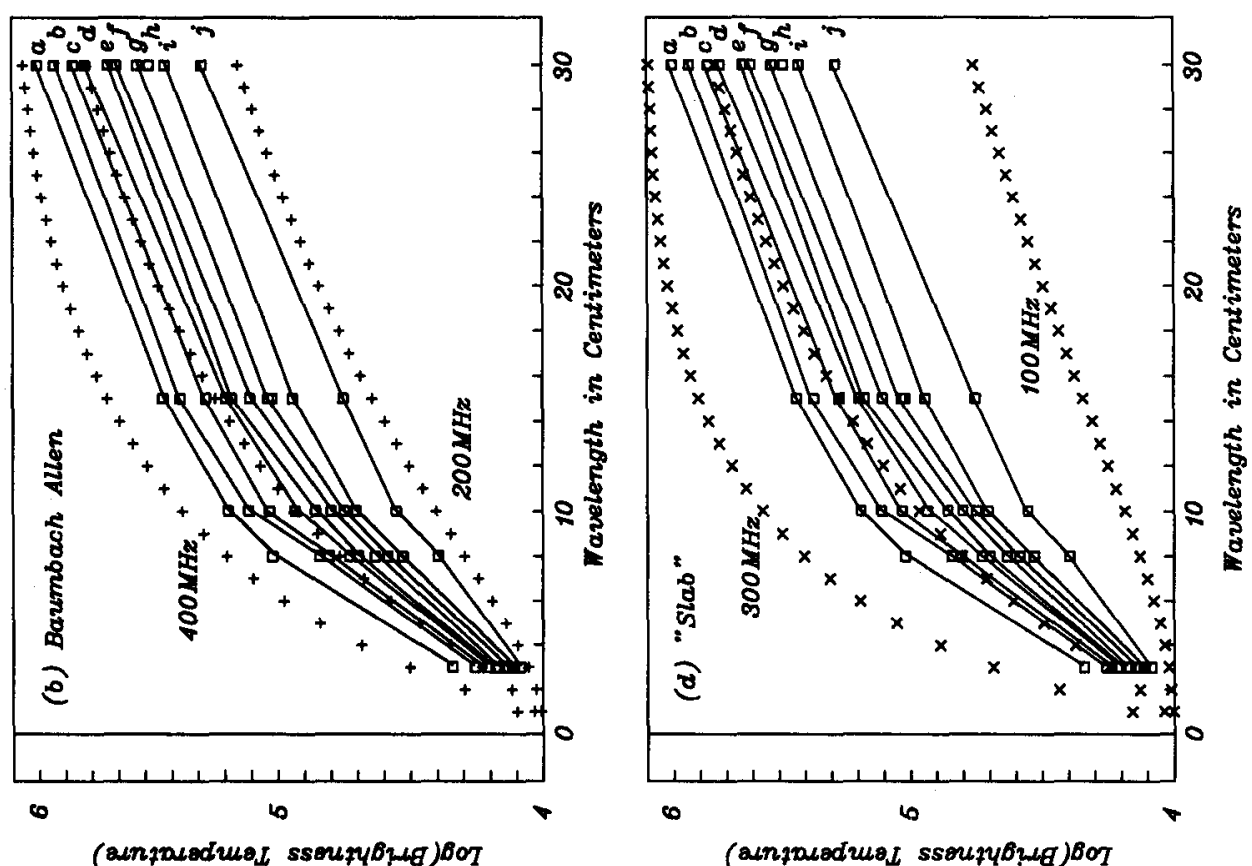

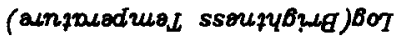

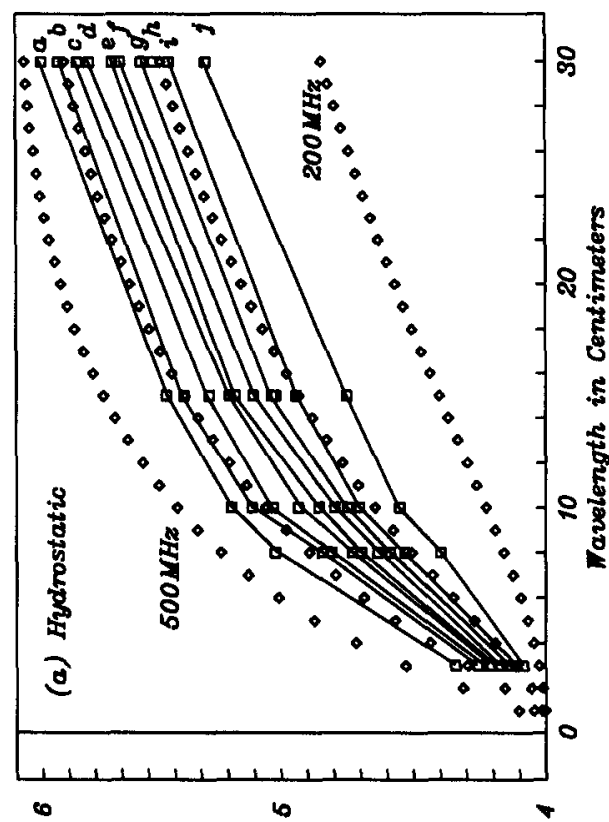

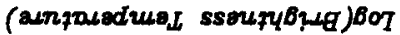

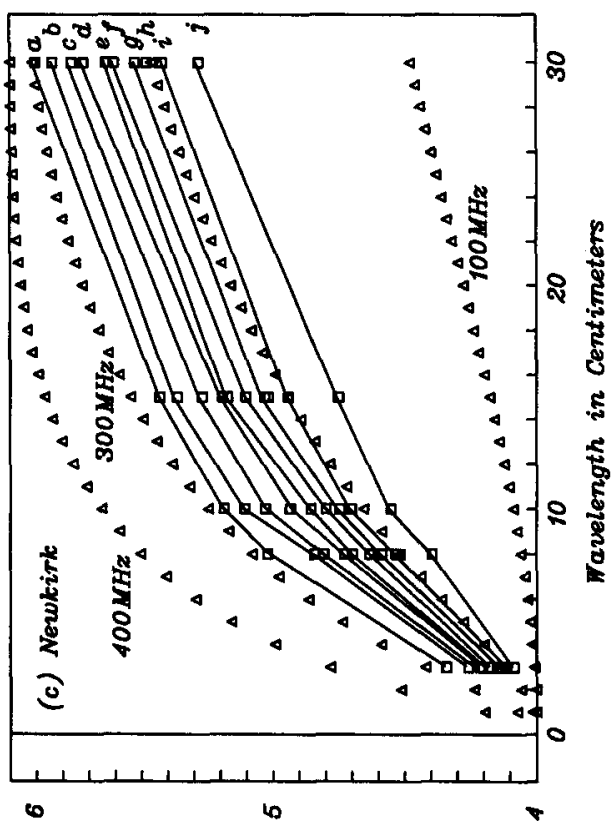

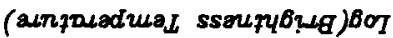

FIGURE 3. Plots of observed solar microwave spectra against those predicted assuming free-free thermal emission and four density models (a: hydrostatic model, b: Baumbach Allen, c: Newkirk, and d: slab). The observed spectra were recorded on (a) $1957 / 10 / 19$, (b) $1957 / 06 / 09$, (c) $1960 / 06 / 30$, (d) $1959 / 12 / 14$, (e) $1960 / 12 / 04$, (f) $1961 / 08 / 11$, (g) $1960 / 11 / 27$, (h) $1961 / 12 / 01$, (i) $1964 / 07 / 27$. 


\subsection{Calculating the brightness temperature}

The brightness temperature is calculated by assigning an initial brightness temperature of $10,000 \mathrm{~K}$ (the chromospheric temperature at the base of the transition region), and integrating the brightness temperature outwards along a vertical ray path through the corona, which is assumed to be isothermal, with an electron temperature of $10^{6} \mathrm{~K}$. If the the refractive index is imaginary at the start of the integration, the brightness temperature is set equal to the coronal temperature at the height where the refractive index first becomes real.

For the density models, we assign a plasma frequency $\left(f_{00}\right)$ at the base of the corona. It is related to the electron density $N_{0}$ by the relation $N_{0}=f_{00}^{2} / 81$. We assume that the corona starts at $10,000 \mathrm{~km}$ above the photosphere.

We compare the results with measurements of the solar flux density at $3,8,10,15$ and $30 \mathrm{~cm}$ wavelength during Cycle 19, published in Nicolet \& Bossy (1984). The values were chosen to cover different parts of the falling phase of the cycle. Other than that, the dates were chosen randomly. Figure 3 shows the observed spectra of solar disc emission, together with the spectra predicted by the free-free emission model for the various atmospheric density models. The frequencies on the plots identify the plasma frequencies $\left(f_{00}\right)$ at the base of the corona used in the density models. We assume that $f_{00}$ is a function of solar activity.

The observed data well fit the free-free emission model of all the density distributions used.

The "quiet Sun" flux densities are extrapolated values corresponding to the minimum of solar activity. Estimates of these are 255, 74, 66, 64, 49, 46 and 41 solar flux units at $3,8,10,10.7,15,21$ and $30 \mathrm{~cm}$ wavelength respectively. If for each density model we pick the value of the $f_{00}$ producing a flux density of 64 solar flux units at $10.7 \mathrm{~cm}$, the errors in the predicted flux densities is better than $10 \%$ for all density models.

The intensity and spectrum of the total microwave emission from the non-flaring Sun is relatively independent of the density model used, but sensitive to the density at the base of the corona, where most of the optical thickness is located.

\section{Discussion and conclusions}

The total centimeter-wavelength emission from the solar disc contains contributions from an often large number of sources. In general the sources are located in active regions, although there is evidence of a weak, widely-distributed emission from outside them. A number of radiative processes are involved, but the dominant one is probably thermal, free-free emission from density concentrations supported in the corona by magnetic fields. Thermal gyroresonance is the main explanation for the bright, compact sources which are often observed close to sunspots. There is evidence for a non-thermal contribution, but if present, it is a probably a minor component which need not be included in models for the total flux.

The correlation between the total disc emission at $10.7 \mathrm{~cm}$ wavelength (the $10.7 \mathrm{~cm}$ flux) and the total magnetic flux over the solar disc, which has been observed to persist for more than a solar cycle, is particularly striking. Only the flux in elements stronger than about 25 Gauss seems to be associated with the emission at $10.7 \mathrm{~cm}$. The total magnetic flux in weaker elements remains reasonably constant over the solar cycle, and shows little correlation with the $10.7 \mathrm{~cm}$ flux.

The correspondence between the total disc emission, which we assume to be predominantly free-free thermal emission, and the total magnetic flux is probably simple. The 
more coronal magnetic flux, the more plasma is supported by it, producing in turn more free-free emission. The contribution by gyroresonance is more direct. The more magnetic flux in the corona, the higher the probability that areas will contain strong enough magnetic fields for gyroresonance to occur at centimeter wavelengths.

The free-free emission from the solar corona is remarkably insensitive to the density models used. It is largely controlled by the density at the base of the corona. This simple connection might account for the good correspondence between the $10.7 \mathrm{~cm}$ flux (for example) and other activity indices. However, the strong dependence of all manifestations of solar activity upon the distribution and movement of magnetic flux, which are in turn driven by the "magnetic activity engine" located below the photosphere might mean that no matter what the mix of emission processes contributing to the total microwave emission from the solar disc might be, there will be a correlation with other emissions driven by the same "magnetic engine".

The observational and theoretical evidence supports a tight, unambiguous relationship between the variations in coronal magnetic fields and the total microwave emission from the solar disc, at least at wavelengths between 2.8 and $21 \mathrm{~cm}$. There has been a considerable amount of research into the localized sources of centimetric emissions in active regions. There is a need for more study of the integrated centimetric emission from the solar disc, and the various sources as contributors to the whole. The total microwave emission from all sources on the solar disc provides a powerful tool for studying the Sun as a magnetic star.

Acknowledgments. The National Solar Observatory/Kitt Peak data used here are produced cooperatively by the National Solar Observatories/National Optical Astronomical Observatory, the National Aeronautical and Space Administration/Goddard Space Flight Center, and the National Atmospheric and Oceanic Administration/Space Environment Laboratory. One of us (KLH) was supported in this work by NASA Contract NASW-4721, while a visitor at the National Solar Observatory (The National Solar Observatories are operated by the Association of Universities in Research in Astronomy Inc. (AURA), in cooperative agreement with the National Science Foundation). We would like to thank O.R. White and R.F. Donnelly for suggestions and comments which helped form the content of this paper, and I. Burke for proof reading this paper a number of times and making some very useful suggestions. Thanks are also due to the Referee, who contributed some valuable points.

\section{REFERENCES}

Alissandrakis, C.E., Kundu, M.R. \& Lantos, P. 1980 A model for sunspot-associated emission at 6-cm wavelength. Astronomy and Astrophysics 82, 30-40.

Alissandrakis, C.E. \& KUndu, M.R. 1984 Center-to-limb variation of a sunspot-associated microwave source. Astron. Ap. 139, 271-284.

Burke, I. 1993 Imaging the Sun with the DRAO Synthesis Telescope. MSc thesis, University of British Columbia, Vancouver, Canada.

Chiuderi Drago, F. \& Melozzi, M. 1984 Non-thermal radio sources in solar active regions. Astronomy and Astrophysics 131, 103-119.

Chiuderi Drago, F., Bandiera, R., Falciani, R., Antonucci, E., Lang, K.R., Willson, R.F., ShibasaKI, K. \& Slottje, C. 1982 Multiple wavelength observations of a solar active region. Solar Physics 80, 71-85.

Christiansen, W.N. \& Mathewson, D.S. 1959 The origin of the slowly-varying component. Paris Symposium on Radio Astronomy (ed. Bracewell). pp. 108-117. Stanford University, Stanford, California. 
Covington, A.E. 1947 Microwave solar noise observations during the partial eclipse of November 23, 1946. Nature 159, 405-406.

Covington, A.E. 1948 Solar noise observations on $10.7 \mathrm{~cm}$. Proceedings of the IRE 36, 454-457.

Denisse, J.F. 1948 Etude des émissions radioeléctriques solaires d'origine purement thermique. $\mathrm{PhD}$. thesis, University of Paris, France.

Erskine, F.T., Kundu, M.R. \& RAO, A.P. 1983 6-cm observations of a solar active region and bursts with 6 seconds resolution. Astronomy and Astrophysics 83, 256-260.

Felli, M., Poletto, G. \& Tofani, G. 1977 Magnetic and microwave structure in solar active regions. Solar Physics 51, 65-81.

Felli, M., LANG, K.R. \& WILlson, R.F. 1981 VLA observations of solar active regions 1, The slowly-varying component. Astrophys. J. 247, 325-327.

GaIZAUSKAS, V. \& TAPPING, K.F. 1980 Long-lived microwave pulsations observed in a complex solar active region. Astrophys. J. 241, 804-810.

Gaizauskas, V. \& TAPPING, K.F. 1988 Compact sites of microwave emission at 2.8 centimeter wavelength inside solar active regions. Astrophys. J. 325, 912-926.

HARVEY, K.L. 1992 Measurements of solar magnetic fields as an indicator of solar activity evolution. In Proceedings of the Workshop on the Solar Electromagnetic Radiation Study for Solar Cycle 22 (ed. R.F. Donnelly). pp. 113-129. NOAA ERL SEL, Boulder, CO., USA.

KAKINUMA, T. \& SWARUP, G. 1962 A model for the sources of the slowly-varying component of microwave solar radiation. Astrophys. J. 136, 975-994.

Koutchmy, S., Zirker, J.B., Steinolfson, R.S. \& Zhugzda, J.D. 1991 Coronal activity. In The Solar Interior and Atmosphere (ed. A.N. Cox, W.C. Livingston \& M.S. Matthews). pp. 1044-1086. Univ. Arizona Press, Tucson, AZ, USA.

KRÜGer, A. 1979 Introduction to Solar Radio Astronomy and Radio Physics. Geophysics and Astrophysics Monographs, No. 6, Vol. 16, D. Riedel Hingham, Mass.

Krüger, A., Hildebrandt, J. \& Furstenberg, F. 1985 A working model for the solar S-component radio emission. Astronomy and Astrophysics 143, 72-76.

Kundu, M.R. 1965 Solar Radio Astronomy. Wiley-Interscience, New York.

Kundu, M.R., Schmahl, E.J. \& RAO, A.P. 1981 VLA observations of solar active regions at 6-cm wavelength. Astron. Ap. 94, 72-79.

LANTos, P. 1968 A model for magnetic gyromagnetic radioemission from solar active regions. Ann. Astrophys 31, 105-113.

LeHANEY, F.J. \& YABSLEY, D.E. 1949 Solar radiation at $1200 \mathrm{Mc} / \mathrm{s}, 600 \mathrm{Mc} / \mathrm{s}$ and $200 \mathrm{Mc} / \mathrm{s}$. Aust. J. Sci. Res., Ser. A 2, 48-62.

NiColet, M. \& Bossey, L. 1984 Aeronomica Acta: "Solar fluxes as indices of solar activity". published by the Belgisch Instituut voor Ruimte Aeronomie, A-No. 282.

Pallavicini, R., Vaiana, G.S., Tofani, G. \& Felli, M. 1979 The coronal atmosphere above solar active regions: the comparison of high spatial resolution soft $\mathrm{X}$-ray and centimetric observations. Astrophys. J. 229, 375-386.

Rabin, D.M., DeVore, C.R., Sheeley, Jr. N.R., Harvey, K.R. \& Hoeksema, J.T. 1991 The solar activity cycle. The Solar Interior and Atmosphere (ed. A.N. Cox, W.C. Livingston \& M.S. Matthews). pp. 781-843. Univ. Arizona. Press, Tucson, AZ, USA.

SHEVGAONKAR, R.K. \& KUNDU, M.R. 1985 Time variability of solar active regions at centimeter wavelengths. Solar Physics 98, 119-126.

Shibasaki, K., Chiuderi Drago, F., Melozzi, M., Slottje, C. \& Antonucci, E. 1983 Microwave, ultraviolet and soft X-ray observations of Hale Region 16898. Solar Physics 89, 307-321.

SlotTJE, C. 1978, Millisecond microwave spikes in a solar flare. Nature 257, 520-521.

Slottje, C. 1980 Fast, fine structure in solar microwave bursts. In Radio Physics of the Sun (ed. M.R. Kundu \& T. Gergely). IAU Symposium 86, 195-201.

SMERD, S.F. \& WESTFOLD, K.C. 1949 The characteristics of radiofrequency radiation in an ionized gas, with applications to the transfer of radiation in the solar atmosphere. Philos. 
Mag. Ser. $740,831-848$.

Tapping, K.F., Kuijpers, J., Kaastra, J.S., van Nieuwkoop J., Graham, D. \& Slottje, C. 1983 VLBI of solar flares. Astronomy and Astrophysics 122, 177-180.

TAPPING, K.F. 1987 Recent solar radio astronomy at centimeter wavelengths, the temporal variability of the $10.7 \mathrm{~cm}$ flux. J. Geophys. Res. No. D1 92, 829-838.

Tapping, K.F. \& DeTracey, B. 1980 The origin of the $10.7 \mathrm{~cm}$ flux. Solar Physics 127, 321332.

Waldmeier, M. \& Müller, K. 1950 Die Sonnenstrahlung im Gebeit von $\lambda=10 \mathrm{~cm}$. Z. für Astrophys. 27, 58-63.

WALDMEIER, M. 1959 A comparison between radioheliograms and optical observations of the solar corona. In Paris Symposium on Radio Astronomy (ed. Bracewell). pp. 118-122. Stanford University, Stanford, California.

Webb, D.F., Davis, J.M., Kundu, M.R. \& Velusamy, T. 1983 X-ray and microwave observations of active regions Solar Physics 85, 267-283.

Zhao, R. \& Jin, S. 1981 C.A.S.: Astronomy Circular \#9. Chinese Astron. Soc. Astron. Circ. 9, 3-7.

ZHELEZnaKov, V.V. 1962 On the origin of the slowly-varying component of the solar radio emission. Soviet Astronomy 6, 3-9.

ZLOTNIK, E.Y. 1968 On the theory of the slowly-varying component of the solar radio emission. Astron. Zh. 45, 310-317.

ZWAAN, C. 1987 Elements and patterns in the solar magnetic field. Annual Reviews of Astronomy and Astrophysics 25, 83-111. 